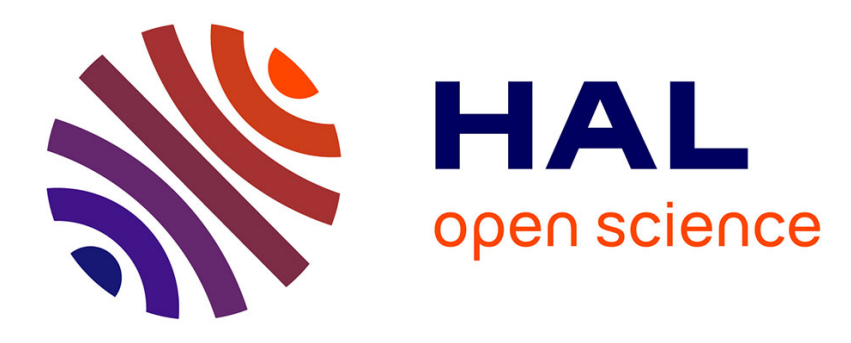

\title{
Influence of Grain Size Distribution and Shape on GPR Waves -Study of Aeolian Dunes
}

\author{
J Guillemoteau, Maksim Bano
}

\section{To cite this version:}

J Guillemoteau, Maksim Bano. Influence of Grain Size Distribution and Shape on GPR Waves -Study of Aeolian Dunes. 72nd EAGE Conference \& Exhibition incorporating SPE EUROPEC 2010, Jun 2010, Barcelona, Spain. hal-01342652

\section{HAL Id: hal-01342652 \\ https://hal.science/hal-01342652}

Submitted on 11 Jul 2016

HAL is a multi-disciplinary open access archive for the deposit and dissemination of scientific research documents, whether they are published or not. The documents may come from teaching and research institutions in France or abroad, or from public or private research centers.
L'archive ouverte pluridisciplinaire HAL, est destinée au dépôt et à la diffusion de documents scientifiques de niveau recherche, publiés ou non, émanant des établissements d'enseignement et de recherche français ou étrangers, des laboratoires publics ou privés. 


\title{
Influence of Grain Size Distribution and Shape on GPR Waves - Study of Aeolian Dunes
}

\author{
J. Guillemoteau* (Institut de Physique du Globe de Strasbourg) \& M. Bano \\ (Institut de Physique du Globe de Strasbourg)
}

\section{SUMMARY}

Ground penetrating radar (GPR), a geophysical method based on electromagnetic (EM) wave propagation, can provide very detailed and continuous images of the internal structures of aeolian dunes. In order to model and explain the origin of observed reflections, we build a model of electric permittivity which accounts for the grain size distribution and shape. By modelling the propagation of GPR waves in frequency domain, we have shown that grain size and grain shape have an influence on GPR reflectivity and we confirmed it by adjusting the modelled data to the real GPR data acquired in arid zones. 


\section{Introduction}

Ground penetrating radar (GPR), a geophysical method based on electromagnetic (EM) wave propagation, can provide very detailed and continuous images of the internal structures of aeolian dunes (Bristow et al., 1996; Bano and Girard, 2001). In these studies, change of water content is believed to be the origin of reflections within the aeolian dunes. However, the present day dunes are located on arid zones and the probability to observe change of the humidity in the first few meters of the dune is very low. On the other hand, some studies have shown that the properties which might change are the grain size distribution (Watson, 1986; Thomas, 1988; Lancaster, 1989 or Wang, 2003) and/or the grain shape (Sen et al., 1981) in the case of a sand/sandstone contact. These two structural parameters are not able to cause a big change of permittivity. However, since dry earth materials have a small relative permittivity (between 2 and 6), the formula of the GPR wave reflectivity suggests that small changes of permittivity are able to cause a strong variation of the reflection coefficient.

First, we propose to explain these reflections by using a model of dry materials in which we can vary the grain size distribution. We developed a method to predict the permittivity of a mix of two grain sizes depending on their respective volumetric fraction. Afterwards, in order to model the reflectivity of GPR waves due to the change of grain shape, we simulated a sand/sandstone contact.

Finally we computed the propagation and the reflection of GPR waves for a two layers model varying the grain size distribution and/or the grain shape. The results of the forward modelling using reasonable geological predictions are in good accordance with real data acquired on present day dunes of the Chadian desert.

\section{Influence of the grain size and grain shape on the electric permittivity of sands}

The propagation and the reflectivity of GPR waves depend on the electric permittivity of the medium. The permittivity of a mix $\left(\kappa_{n}\right)$ of several grains sizes (of permittivity $\kappa_{q}$, quartz in our case) can be computed by the recursive formula introduced by Robinson and Friedman (2001):

$$
\kappa_{n}=\kappa_{n-1}+3 \kappa_{n-1} \frac{f_{n}}{\phi+\sum_{n=1}^{n} f_{n}} \cdot \frac{\left(\kappa_{q}-\kappa_{n-1}\right)}{\kappa_{q}+2 \kappa_{n-1}-\frac{f_{n}}{\phi+\sum_{n=1}^{n} f_{n}}\left(\kappa_{q}-\kappa_{n-1}\right)}
$$

$f_{n}$ is the volumetric fraction of the grains of size " $n$ " which has to validate the condition:

$$
\sum_{n=1}^{N} f_{n}=1-\phi
$$

Where $N$ is the total number of sizes and $\phi$ is the porosity. Generally, the data from granulometry are given in percentage of weight. If $A_{n}$ is the percentage of weight of the size " $n$ ", the volumetric fraction of the present grain size is given by:

$$
f_{n}=(1-\phi) \frac{A_{n}}{\sum_{1}^{N} A_{n}}
$$

This model depends on the porosity of the medium. However, one has to keep in mind that the porosity depends also on the grain size distribution. Thus, the two parameters $f_{n}$ and $\phi$ are joined, and we suggest to compute, first, a realistic porosity associated to the grain size distribution. It is very difficult to predict the porosity for a mix of more than two sizes. We used the empirical "fractional packing model” (Koltermann and Gorelick, 1995) which is adapted to bimodal mixtures. Therefore, in order to predict the permittivity using the right porosity, we propose to combine the formula (1) and the fractional packing model as it is shown in figure 2. 


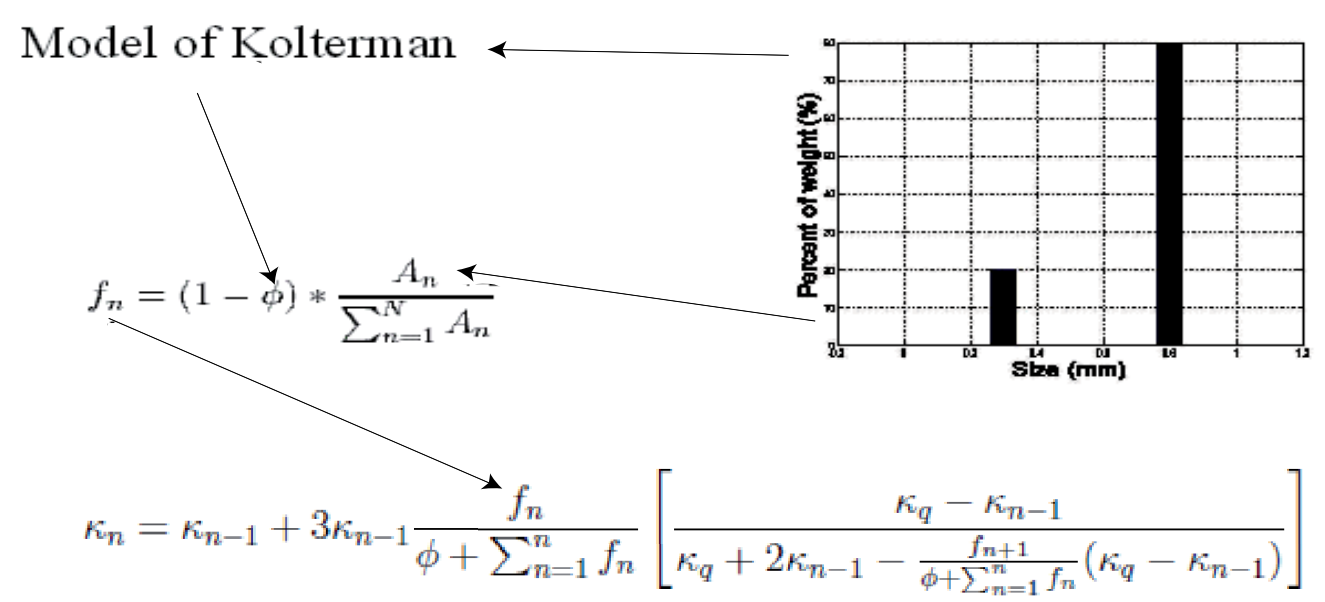

Figure 1 Schematic presentation showing how to predict the permittivity from the data of granulometry. $A_{n}$ is the grain size distribution.

The variation of the permittivity due to the change of grain shape can be predicted by the formula (Sen et al., 1981):

$$
\left[\frac{\kappa_{q}-\kappa^{*}}{\kappa_{q}-\kappa_{0}}\right]\left[\frac{\kappa_{0}}{\kappa^{*}}\right]^{L}=\phi
$$

The parameter $\mathrm{L}$ is called depolarisation factor and depends on the grain shape (Stratton, 1941). Its value varies between 0 and 1 .

\begin{tabular}{|c|c|c|c|}
\hline Shape & Name & Axes & Depolarising factor \\
\hline \hline & Cylinder & $a=b<<c$ & $\mathrm{~L}=0$ \\
\hline 0 & Sphere 'Prolate' & $a=b<c$ & $0<L<1 / 3$ \\
\hline A & Sphere & $a=b=c$ & $L=1 / 3$ \\
\hline & Sphere 'Oblate' & $a=b>c$ & $1 / 3<L<1$ \\
\hline & Disk & $a=b>c$ & $L=1$ \\
\hline
\end{tabular}

Table 1 Depolarising factor for different kind of grain shape.

\section{Modelling of GPR reflections observed inside an aeolian dry sand dune}

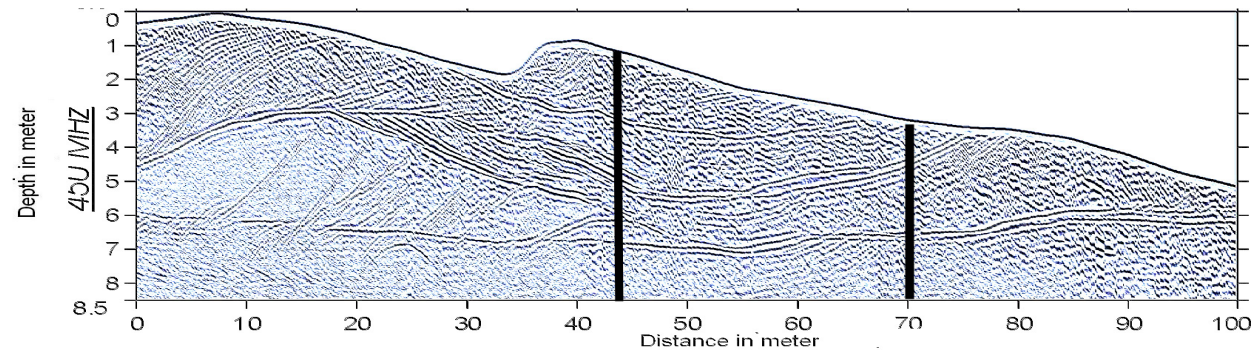

Figure 2 GPR section acquired over an aeolian dune with an antenna of $450 \mathrm{MHz}$. The two vertical black lines refer to the traces which have been studied.

A GPR profile (Fig. 2) acquired on a dry dune in Chadian desert shows many strong reflections in its interior. Owing to the dry climate of the area (arid zone) and also to the geometry of the reflections (which are not flat), one can think that these reflections have no chance to be caused by changes of the water content inside the dunes. We modelled the first reflection of the interior of the dune considering 
a change of grain size distribution. We considered a two layers model in which the permittivity has been computed using small grains for the first layer and a mix of small and large grains (with volumetric fraction of $20 \%$ and $80 \%$, respectively) for the second layer. The porosity of the two layers is calculated by the fractional packing model described above. This gives a porosity of 0.4 for the first layer and 0.24 for the second one. The resulting permittivity for the two sands is between 2 and 3, which is in good agreement with values given in the literature (e.g. Davis and Annan, 1989).

The GPR response related to this model has been computed by using a 1D modelling method in frequency domain (Bano and Girard, 2001; Bano, 2004). These synthetic data are computed for a large number of arrival time (propagation time) and compared to the measured signals. The right propagation time is matched by minimizing the error between the synthetic and real data. The comparison between modelled and measured data is presented in figure 3 . The real signals are from traces pointed out by two black lines on figure 2 .
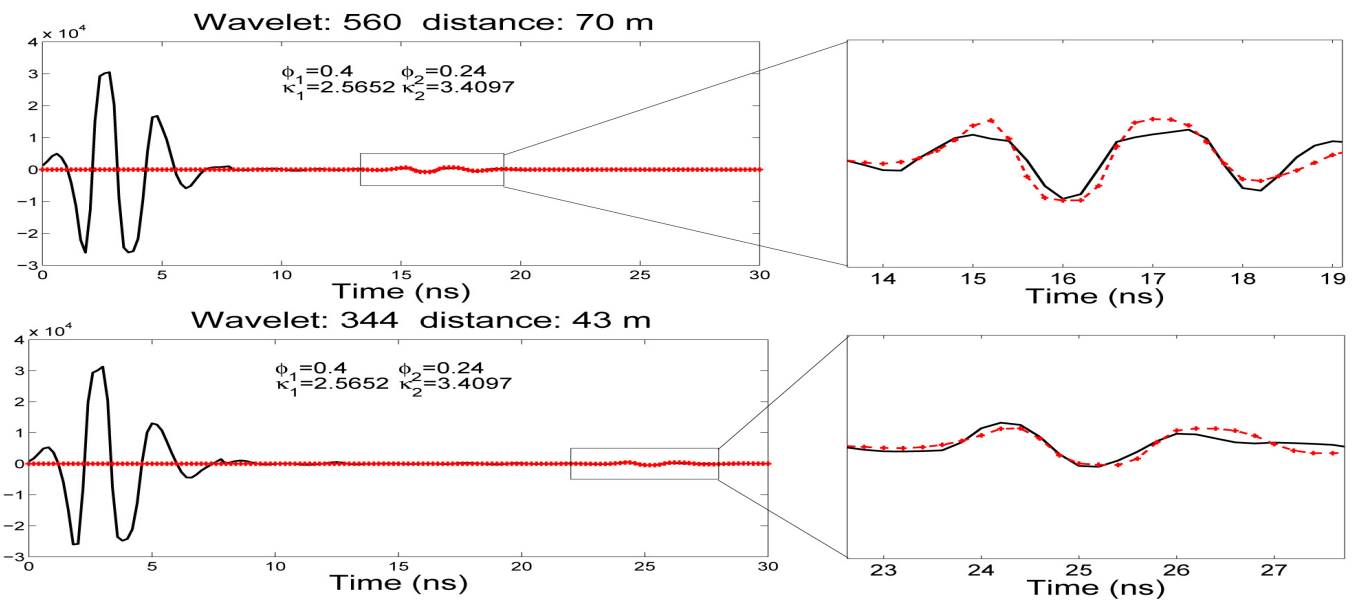

Figure 3 Comparison between modelled (in red) and observed (in black) data for a contact between two sands with different grain size distribution. The real wavelets are located at $x=70 \mathrm{~m}$ and $x=43 \mathrm{~m}$ on the section of figure 2.

\section{Modelling of GPR reflections observed from a sand/sandstone contact}

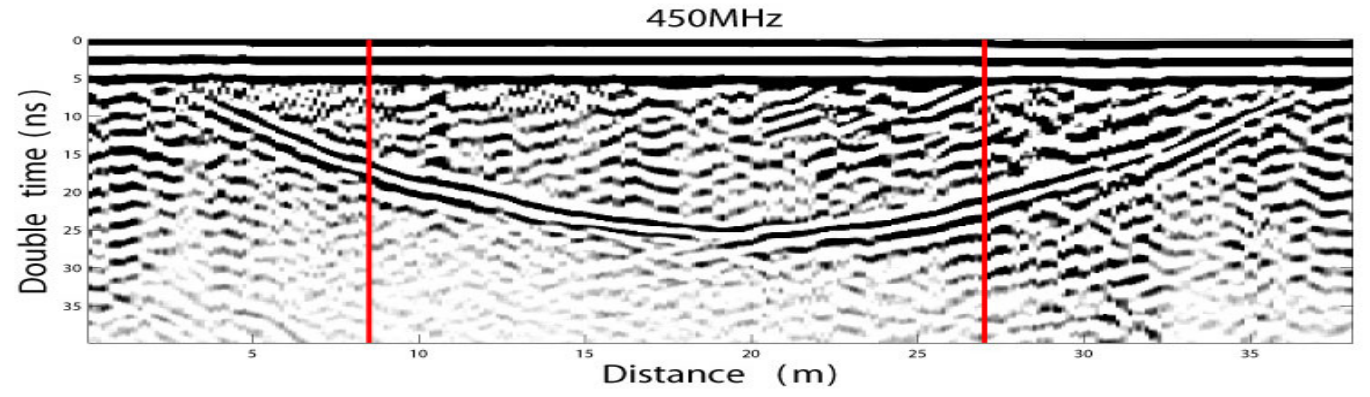

Figure 4 GPR section acquired over an aeolian dune with an antenna of $450 \mathrm{MHz}$. The bent reflection is the base of the dune. The two vertical lines refer to the wavelets which have been studied.

The same experience has been performed in order to interpret the GPR data acquired on a sand/sandstone contact. Figure 4 shows GPR data (without topographic corrections) acquired on a dry aeolian dune whose base (the bent reflection) is constituted of sandstone. We computed the reflectivity from the base of the dune (sand/sandstone contact) using the formula (4). We modelled the sand by a layer composed of spherical grains $(\mathrm{L}=0.33)$ with a porosity equal to 0.36 , while the sandstone is considered as a medium composed of oblate grains $(\mathrm{L}=0.5)$ with a lower porosity equal to 0.1 . The results of this modelling for the two soundings, shown by two red lines in figure 4, are presented in figure 5 . 


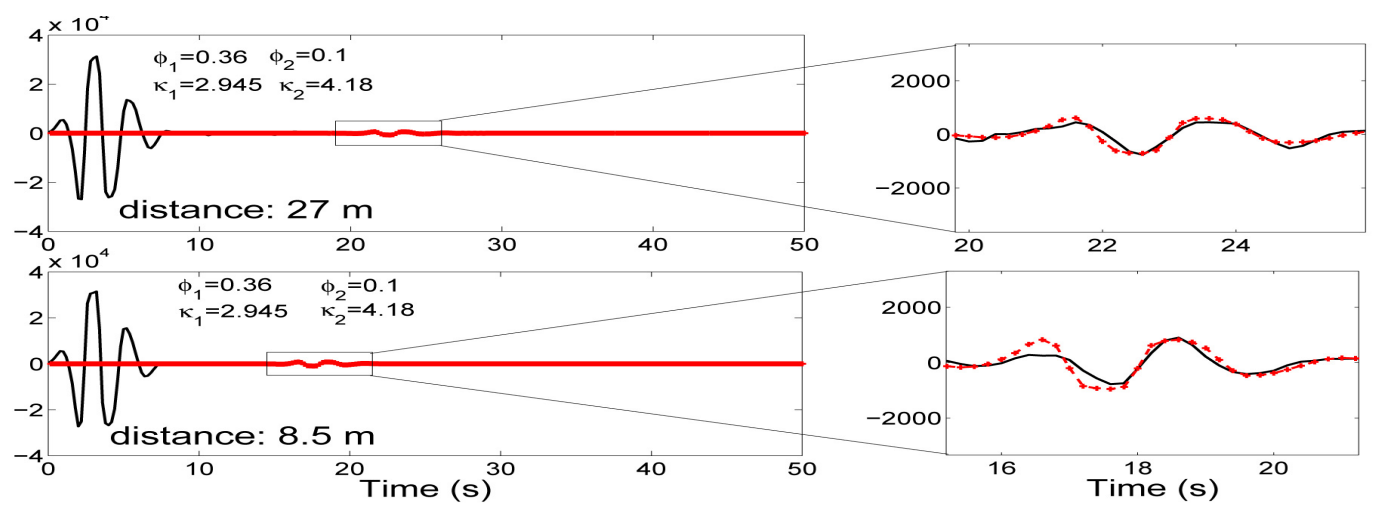

Figure 5 Comparison between modelled (in red) and observed (in black) data for a sand/sandstone contact. The real wavelets are located at $x=27 \mathrm{~m}$ and $x=8.5 \mathrm{~m}$ on the section of figure 4 .

\section{Conclusions}

In this study, we tried to relate physics parameters (like grain size or shape) to the reflectivity of GPR waves. By using analytical models to compute the permittivity, we have shown that grain size and grain shape have an influence on GPR reflections and we confirmed it by adjusting the modelled data to the real GPR data acquired in arid zones. This kind of modelling is restricted to the case of dry media in which changes of grain size distribution or shape induces small change on the permittivity, but enough variations on the reflectivity. In addition, the presence of the water (that causes strong change on the permittivity) might hide the effects of the granular composition of the media. However, by this study, we show that one has to integrate these effects to model the GPR data in which water content variations and change of lithology are combined. Extending the application of the proposed method to radar data acquired on controlled dry materials in laboratory is the logical next step.

\section{References}

Bano, M. and J.-F. Girard, 2001. Radar reflections and water content estimation of Aeolian sand dune, Geophysical Research Letter, 28, 3207-3210.

Bano, M., 2004. Modelling of GPR waves for lossy media obeying a complex power law of frequency for dielectric permittivity: Geophysical Prospecting, 52, 11-26.

Bristow, C., J. Pugh, and T. Goodall, 1996. Internal structure of Aeolian sand dunes in Abu Dhabi determined using ground penetrating radar, Sedimentology, 43,995-1003.

Davis, J. and A. Annan, 1989. Ground-penetrating radar for high resolution mapping of soil and rock stratigraphy, Geophysical Prospecting, 37, 531-551.

Koltermann, C. and S. Gorelick, 1995. Fractional packing model for hydraulic conductivity derived from sediment mixtures, Water Resources research, 31, 3283-3297.

Lancaster, N., 1989. The Namib sand sea: Dunes forms, processes and sediments: Rotherdam, 200 pp.

Robinson, D. and S. Friedman, 2001. Effect of particle size distribution on the effective dielectric permittivity of saturated granular media, Water Resources Research, 37, 33-40.

Sen, P., C. Scala, and M. Cohen, 1981. A self-similar model for sedimentary rocks with application to dielectric constant of fused glass beads, Geophysics, 46, 781-795.

Stratton. J. A., 1941. Electromagnetic theory, New York. McGraw-Hill Book Co. Inc.

Thomas, D. S. G., 1988. Analysis of linear dune sediment-form relationships in the Kalahari dune desert: Earth Surface Processes and Landforms, 16, 545-553.

Wang X., Z. Dong, J. Zhang, J. Qu and A. Zhao, 2003. Grain size characteristics of dune sands in the central Taklimakan Sand Sea: Sedimentary Geology, 161, 1-14.

Watson, A., 1986. Grain size variations on a longitudinal dune and a barchan dune: Sedimentary Geology, 46, 49-66. 